\title{
Monsieur Lucien Otis : type du prêtre-colonisateur, aux origines du Saguenay (1856-1867)
}

\section{Raoul Lapointe}

Volume 55, 1988

Cent cinquante ans d'histoire religieuse au Saguenay-Lac-Saint-Jean (1838-1988)

URI : https://id.erudit.org/iderudit/1006946ar

DOI : https://doi.org/10.7202/1006946ar

Aller au sommaire du numéro

Éditeur(s)

Les Éditions Historia Ecclesiæ Catholicæ Canadensis Inc.

ISSN

0318-6172 (imprimé)

1927-7067 (numérique)

Découvrir la revue

Citer cet article

Lapointe, R. (1988). Monsieur Lucien Otis : type du prêtre-colonisateur, aux origines du Saguenay (1856-1867). Sessions d'étude - Société canadienne d'histoire de l'Église catholique, 55, 49-62. https://doi.org/10.7202/1006946ar
Résumé de l'article

M. Lucien Otis naquit à la Baie St-Paul en 1824 et fit ses études àSte-Anne-de-la-Pocatière. Associé de près au développement du Saguenay, il a laissé son nom à un lac, à un village et à un canton. D'abord curé de

St-Alphonse de Bagotville puis de l'Anse-St-Jean, la maladie l'obligea àlaisser sa terre d'adoption après y avoir consacré onze ans de sa vie. Il mourut à Québec le 12 juin 1868. Il tomba alors dans un profond oubli qui devait durer plus d'un siècle. Jusqu'à tout récemment, seuls les documentsd'archives et les cartes géographiques conservaient sa mémoire. Ce prêtre modèle qui représente le type du curé d'autrefois méritait un meilleur sort et une publication récente vient rendre justice à cet humble pionnier de la région du Saguenay. Le présent article donne un aperçu de cet ouvrage intitulé : LE CAHIER DE MONSIEUR OTIS.
Tous droits réservés @ Les Éditions Historia Ecclesiæ Catholicæ Canadensis Inc., 1988
Ce document est protégé par la loi sur le droit d'auteur. L'utilisation des services d'Érudit (y compris la reproduction) est assujettie à sa politique d'utilisation que vous pouvez consulter en ligne. 


\title{
Monsieur Lucien Otis: type du prêtre-colonisateur, aux origines du Saguenay (1856-1867)
}

\author{
Raoul LAPOINTE, historien \\ Chicoutimi, Québec
}

\section{RÉSUMÉ DE LA CONFÉRENCE}

M. Lucien Otis naquit à la Baie St-Paul en 1824 et fit ses études à Ste-Anne-de-la-Pocatière. Associé de près au développement du Saguenay, il a laissé son nom à un lac, à un village et à un canton. D'abord curé de St-Alphonse de Bagotville puis de l'Anse-St-Jean, la maladie l'obligea à laisser sa terre d'adoption après y avoir consacré onze ans de sa vie. Il mourut à Québec le 12 juin 1868. Il tomba alors dans un profond oubli qui devait durer plus d'un siècle. Jusqu'à tout récemment, seuls les documents d'archives et les cartes géographiques conservaient sa mémoire. Ce prêtre modèle qui représente le type du curé d'autrefois méritait un meilleur sort et une publication récente vient rendre justice à cet humble pionnier de la région du Saguenay. Le présent article donne un aperçu de cet ouvrage intitulé: LE CAHIER DE MONSIEUR OTIS.

Charles Trenet a écrit une magnifique chanson sur l'oubli:

Longtemps, longtemps, longtemps après que les poètes ont disparu

Leurs chansons courent encore dans les rues

La foule les chante un peu distraite en ignorant le nom de l'auteur

Ainsi en est-il non seulement des poètes mais de tous ceux qui, à un moment donné ont brillé dans un domaine quelconque. Les plus grands génies, les rois, les conquérants passent par l'oubli et, après un certain temps, on se rappelle à peine ces noms autrefois si célèbres; on doit s'aider d'un dictionnaire pour se rafraîchir la mémoire. L'exemple de Champlain est d'actualité. On sait qu'il a fondé Québec et qu'il a joué un très grand rôle en Nouvelle-France mais on a perdu la trace de son tombeau. Par ailleurs, combien de musiciens aujourd'hui très connus ont été longtemps oubliés. Les deux exemples souvent cités sont ceux de Jean-Sébastien Bach et d'Antonio Vivaldi. Pendant près d'un siècle, Bach fut oublié et c'est en le ressuscitant qu'on découvrit qu'Antonio Vivaldi avait aussi 
existé. Pourtant de leur vivant ces musiciens étaient particulièrement célèbres. On pourrait dire la même chose d'autres artistes, peintres ou écrivains. Qui se souvient d'avoir lu qu'Adolphe-Philippe Dennery, l'auteur des Deux Orphelines, était le maître incontesté du mélodrame au siècle dernier? Il faut visiter, à Paris, le musée qui porte son nom pour se rendre compte de l'importance qu'avait cet auteur aujourd'hui oublié... Sit transit gloria mundi... Oui, ainsi passe la gloire...

M. Lucien Otis qui a vécu de 1824 à 1868 , n'a pas échappé à la règle: lui aussi a passé comme une étoile filante. Ce missionnaire, qui, pendant plus de dix ans, a dépensé sa vie goutte à goutte sur une terre lointaine et sauvage a eu lui aussi son heure de gloire pour retomber peu à peu dans l'oubli. La résurrection de ce pionnier, malade et silencieux, n'a pas été chose facile puisque son nom était absent des dictionnaires, même du prestigieux Dictionnaire biographique du Canada qui, pourtant, essaie de retracer tous ceux qui ont fait leur marque chez nous. Il m'a fallu recourir à de nombreux écrits pour être capable de rassembler les morceaux de ce véritable casse-tête et de pouvoir enfin remettre sur son piédestal cet homme d'envergure qui avait été balayé lui aussi par le vent de l'oubli.

Mon grand-père, Ferdinand Otis, parlait souvent d'un prêtre qui était son parent et auquel il devait, ce qui était assez rare à l'époque, de savoir lire et écrire. Il nourrissait une très grande admiration pour celui qu'il appelait «Monsieur Otis», cet intellectuel qui, pendant dix ans, semble avoir été égaré dans la forêt saguenéenne. Ce prêtre, déjà surchargé par son ministère ordinaire, avait ajouté à sa tâche des heures d'enseignement. Il réunissait un certain nombre de garçons de sa paroisse auxquels il apprenait les rudiments de la lecture et du calcul. Il avait aussi établi des écoles et fondé deux bibliothèques dont une à Saint-Alphonse de Bagotville qui fut la première au Saguenay. C'est ainsi qu'à cette époque où la plupart de nos ancêtres déclaraient dans les actes officiels «ne pas savoir signer», mon grand-père avait pu échapper à cette épidémie d'analphabétisme. Aussi gardait-il un souvenir reconnaissant à «Monsieur Otis» qui était responsable du début d'instruction dont il avait pu bénéficier.

Plus tard, j'appris que le canton Otis, le lac du même nom ainsi que le village qui le surplombe, avaient été ainsi nommés en l'honneur de ce même prêtre qui avait joué un rôle important dans l'ouverture du Saguenay. J'eus même le bonheur un jour de voir un portrait de Monsieur Otis accroché dans le sous-sol de l'église Saint-Alphonse de Bagotville où il avait été l'un des premiers pasteurs.

En parcourant l'ouvrage de Gérard Malchelosse, intitulé «Généalogie de la famille Otis, branche canadienne», je vis que l'auteur avait consacré quatorze lignes à ce pionnier. C'était déjà un traitement de faveur dans cet 
ouvrage de généalogie qui n'est pas très bavard, mais j'aurais aimé quelque chose de plus élaboré. Alors que je poursuivais mes recherches pour en savoir un peu plus sur cet homme, j'appris qu'il existait un livre de prônes rédigé par Monsieur Otis. J'eus vite fait de me procurer une copie de ce manuscrit. Ce document me parut d'abord assez mince pour servir de base à une biographie de ce missionnaire; mais, après l'avoir lu attentivement, je m'aperçus qu'il contenait beaucoup plus d'information qu'il n'en laissait paraitre. En m'aidant de nombreux manuscrits et d'une quantité impressionnante d'ouvrages de toutes sortes, je réussis malgré toute l'humilité de Monsieur Otis, à sortir ce prêtre des murs de l'oubli qu'il avait construits autour de lui.

Je vous donnerai ici un aperçu de ce que fut cet homme qui, en d'autres circonstances, aurait pu être un brillant professeur d'université mais qui s'est contenté de passer dix ans de sa vie, perdu dans la forêt bordant le Saguenay, afin de pouvoir aider de pauvres colons encore plus à plaindre que lui.

Lucien Otis, cet inconnu que seuls les indiscrétions de nos cartes routières, les documents d'archives et les registres de paroisses pouvaient nous faire connaître, jusqu'à tout récemment, était originaire de Charlevoix. Il naquit à la Baie-Saint-Paul le 18 octobre 1824. Il était le fils d'Alexis Otis et de Marie Thibault. Entré au Collège de Sainte-Anne-dela-Pocatière en 1840, il fut ordonné prêtre le 27 juillet 1851.

Lorsqu'on étudie une chronologie comparative mettant en regard la biographie de Monsieur Otis, certains événements et certaines dates qui ont ponctué l'histoire du Saguenay à son début, on s'aperçoit que cet homme était bien préparé pour sa mission. Il était, comme nous venons de le voir, originaire de Charlevoix, terre natale de nos premiers colons; c'était là que s'était formée la Société des 21 pour la colonisation du Saguenay. Dans une petite paroisse comme Baie Saint-Paul, c'était vraisemblablement le sujet du jour. Lucien Otis avait 14 ans au moment de la fondation de la Société des 21 . Il était donc assez âgé pour prendre part aux discussions. Lorsqu'il est ordonné prêtre, en 1851, il devient vicaire dans sa paroisse natale où il est assigné jusqu'en 1856. Il est, durant cinq ans, replongé dans un milieu qui comptait plusieurs compatriotes exilés au Saguenay.

Lucien fait ses études à Sainte-Anne-de-la-Pocatière où il demeure de 1840 à 1851 . Durant ses vacances, qui durent un mois et demi chaque année, il revient à la Baie Saint-Paul; mais pendant ses mois d'études à Sainte-Anne, il peut suivre les projets qu'on échafaude concernant la colonisation du Saguenay. Durant son séjour à La Pocatière, deux grands mouvements voient le jour. En 1847, M. l'abbé Antoine Racine, vicaire à la 
Malbaie et frère du futur Mgr Dominique Racine, premier évêque de Chicoutimi, fonde la «Société de défricheurs et de cultivateurs du Saguenay1", qui deviendra la "Société des défricheurs de la Rivière-auxSables». En 1849, c'est la fondation de l'«Association des comtés de L'Islet et de Kamouraska ${ }^{2}$ », qui est à l'origine du peuplement du lac SaintJean. Lucien Otis est un élève de François Pilote qui, en 1850, fait son célèbre voyage au Saguenay et qui, en 1852, publie un ouvrage non moins célèbre intitulé: Le Saguenay en 1851. Monsieur Otis est donc dans une situation idéale pour être bien informé touchant son futur champ d'action et pour être embrasé du feu sacré qui, quelques années plus tard, devait le propulser dans cette vaste région encore à organiser.

Mais contentons-nous de le suivre à la trace.

M. Lucien Otis fit son entrée au Saguenay en 1856. Voici de quelle façon il parle de cet événement dans son Cahier d'annonces pastorales: «Je suis arrivé à Saint-Alphonse dans la nuit du 18 septembre 1856. Je chantai ma lère messe à Saint-Alphonse le 28 septembre et la lère à

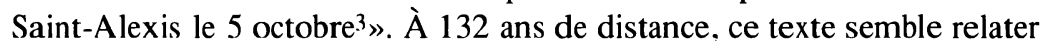
un simple fait divers, qui ne nous émeut guère; mais, si on le place dans son contexte historique, on peut dire que, dès son arrivée, le nouveau curé marche sur des œufs ou, si vous préférez une expression plus moderne, que Monsieur Otis devait s'attendre à devoir patiner.

De nos jours, la distance qui sépare ces deux paroisses représente une quantité négligeable: la route est asphaltée, les véhicules sont rapides et confortables, des ponts modernes enjambent les «deux rivières» qui se jettent dans la Baie; mais au début de la colonisation, ce trajet était tout un défi surtout au moment du dégel et de la crue des eaux. Il aurait été normal que les pionniers se regroupent en un même endroit pour éviter les déplacements inutiles et surtout hasardeux. Mais l'Histoire nous présente une autre réalité.

Dans la région de Charlevoix, il existait depuis fort longtemps une antipathie naturelle entre les habitants de la Baie Saint-Paul et ceux de la Malbaie. Comme il y avait, parmi les premiers colons venus au Saguenay, des représentants de l'un et de l'autre endroit, cette hostilité congénitale fut transportée jusque sur nos rives de sorte que, nous fait remarquer $\mathbf{M}$. Louis-Antoine Martel, «les citoyens venus de Baie Saint-Paul s'établirent

1 Victor Tremblay, Histoire du Saguenay, depuis les origines jusqu'en 1870, Publication No 21 de la Société historique du Saguenay, 1968, p. 330.

2 Ibid., p. 339.

${ }^{3}$ R. Lapointe, Le cahier de Monsieur Otis, Cahiers de Saguenayensia No 1 (Études et documents) 1986, p. 38. 
surtout à Saint-Alphonse et ceux de la Malbaie, à Saint-Alexis4». Cette double concentration des premiers arrivants devait susciter des tiraillements à une époque où l'unité était de mise.

La première chapelle fut construite à Saint-Alexis dès 1841, tandis que celle de Saint-Alphonse ne le fut qu'en 1844. Il était donc historiquement établi que le curé désigné résidait à Saint-Alexis où se trouvait d'ailleurs le gros de la population. La chapelle de Saint-Alphonse n'était qu'une desserte qui, en fait, n'aurait jamais dû exister puisqu'elle venait perturber une vie déjà passablement difficile. Martel reproche au Père Honorat d'avoir fixé une chapelle à Saint-Alphonse:

L'érection de cette chapelle, dit-il, à une si grande proximité de la première, n'était pas un plan bien avisé, d'autant plus que ces deux petites paroisses réunies ensemble n'auraient fait qu'une seule bonne paroisse qui serait devenue indubitablement le chef-lieu du comté, à la place de Chicoutimi qui l'enleva à la Gde Baie quelques années plus tard pendant que les deux paroisses de Saint-Alexis et de Saint-Alphonse se chicanaient à qui le posséderait 5 .

Les Oblats, arrivés au Saguenay en 1844, quittèrent cette région en 1853 et c'est M. Léandre Gill qui les remplaça avec résidence à SaintAlexis comme le voulait la tradition. Il était donc acquis que Saint-Alexis était le pied-à-terre du curé et ce, depuis l'arrivée de M. Charles Pouliot en 1842. On trouvait à cet endroit une chapelle bien organisée, de même qu'une sacristie et un presbytère. Or, en 1854, la population de SaintAlexis décroît et celle de Saint-Alphonse augmente. Deux projets s'élaborent alors pour corriger la situation:

Le premier c'est de construire une chapelle entre les «deux rivières», c'est-à-dire entre la rivière $\mathrm{Ha}$ ! Ha! du côté de Saint-Alexis et la rivière à Mars du côté de Saint-Alphonse. Dès 1845, les Oblats ont même fait l'acquisition d'un terrain mesurant deux acres de largeur «entre les deux rivières» à cette fin. Cette chapelle aurait été située à peu près où se trouve la chapelle Notre-Dame-de-la-Baie non loin de l'église Saint-Édouard de Port-Alfred.

Le second projet voulait que la résidence du curé soit fixée à SaintAlphonse, à cause de sa population grandissante, et que Saint-Alexis ne soit qu'une desserte.

Connaissant le contexte historique, on s'imagine facilement que la population de Saint-Alexis est inquiète et qu'elle sent le tapis lui glisser lentement sous les pieds. Essayant de conserver à la fois la chèvre et le chou, l'évêque tranche la question en 1851 en statuant que Saint-Alexis et

4 L.-A. Martel, Notes sur le Saguenay, Texte manuscrit publié par le Centre d'Études et de Recherche historiques du Saguenay, Chicoutimi, 1968, p. 14.

5 Ibid., p. 45. 
Saint-Alphonse forment deux fabriques distinctes. Mais la population de Saint-Alexis continue de décroître au profit de la paroisse voisine et, le 19 novembre 1854, M. Gill obtient la permission de déménager à SaintAlphonse à condition qu'il puisse assurer la messe à Saint-Alexis tous les dimanches. Ne voulant pas exaspérer la population, il déménage sans attirer l'attention, on ne sait pas exactement à quel moment.

Lorsque M. Otis arrive à Saint-Alphonse en 1856 , la résidence du curé est bel et bien fixée à cet endroit et Saint-Alexis n'est qu'une desserte. Jusque-ià cependant Saini-Alexis pouvait âvoii la messe touis les dimanches puisque M. Gill avait un vicaire. Mais en $1856 \mathrm{M}$. Otis arrive seul pour remplacer ces deux prêtres; car M. Gill est nommé directeur du pensionnat de l'Université Laval et M. Morisset devient curé de SaintFidèle de la Malbaie. M. Lucien Otis est rempli de bonne volonté mais c'est un homme malade et il doit à lui seul accomplir la besogne de ses deux prédécesseurs. M. Louis-Antoine Martel décrit la situation en ces termes:

Monsieur Lucien Otis n'ayant pas de vicaire entreprit de desservir SaintAlphonse et Saint-Ảlexis également c.a.d. à dire deux messes tous les dimanches; il s'aperçut bientôt qu'il ne pourrait résister longtemps et en 1857 avec la permission de l'Évêque, il fut décidé qu'il n'irait faire l'office à Saint-Alexis qu'un seul dimanche sur trois. De là grand mécontentement de la part des citoyens de cette dernière paroisse qui craignait (sic) d'être obligé (sic) de faire partie tôt ou tard de la paroisse de Saint-Alphonse 6 .

Perrmettez-moi de résumer la situation pour qu'on puisse bien imaginer quel devait être le travail inhumain qui attendait $M$. Otis à son arrivée au Saguenay en 1856.

De 1842 à 1856, la population ne cesse d'augmenter dans le secteur que le nouveau curé doit desservir, passant de 2500 à 6000. En 1842, M. Charles Pouliot était nommé missionnaire au Saguenay. Il était seul. En 1844, arrivent les Oblats pour le remplacer: ils sont quatre. À leur départ, en 1853, M. Gill les remplace et, peu de temps après son arrivée, on lui donne un vicaire. En 1856, M. Gill et M. Morisset, son vicaire, quittent le Saguenay et M. Otis remplace les deux. Comme vous voyez, la politique des coupures de personnel ne date pas d'aujourd'hui, sauf qu'en 1856 on ne pouvait mettre cette politique de restriction sur le dos de l'informatique.

Outre le fait que la population ne cesse d'augmenter, M. Lucien Otis recueille dès son arrivée deux problèmes de taille:

D'abord, on l'a vu plus haut, la résidence du curé est maintenant à Saint-Alphonse et il doit desservir adéquatement la population de SaintAlexis, qui l'attend d'un pied ferme. En effet, les paroissiens de Saint-

6 Ibid., p. 67. 
Alexis venant en majorité de la Malbaie, doivent se dire: «Le nouveau curé est originaire de la Baie-Saint-Paul et il arrive au milieu des siens puisque les gens de Saint-Alphonse, viennent en général de la Baie SaintPaul. On peut donc s'attendre à ce que Saint-Alexis demeure longtemps une desserte». Outre ces considérations basées sur des problèmes locaux, il reste qu'on ne peut modifier le cours de l'histoire en retranchant un droit acquis sans s'exposer à ramer contre le courant populaire et sans provoquer une levée de boucliers. Qu'on se rappelle les remous que suscita le choix de Constantinople comme capitale de l'Empire romain; qu'on se rappelle aussi le transfert de la résidence du pape de Rome à Avignon de même que la tentative de déplacement d'un certain palais cardinalice au Québec.

Le deuxième problème que doit solutionner $M$. Otis c'est celui de remplacer la chapelle de Saint-Alphonse qui est vétuste et exiguë.

Et n'oublions pas que ce prêtre, dont la santé est aussi délabrée que la chapelle de Saint-Alphonse, doit s'occuper aussi d'un chapelet de petites missions échelonnées sur les deux rives du Saguenay: Tableau, AnseSaint-Jean, Petit-Saguenay, Rivière Sainte-Marguerite qui, à cette époque, sont loin d'être des lieux de villégiature.

Malgré tout cela, le nouveau curé s'attèle à la tâche dès son arrivée. Il est vrai que sa santé laisse à désirer mais il a beaucoup de courage et, ce qui n'est pas à dédaigner, il est intelligent, fin diplomate et il sait manier la plume et l'argumentation. Balzac disait qu'il voulait continuer par la plume ce que Napoléon avait commencé par l'épée. M. Otis aurait pu dire: «Ce que la santé et le nombre ont permis à mes prédécesseurs de réaliser, je vais le continuer par des requêtes».

M. Otis n'est demeuré que cinq ans à Saint-Alphonse, mais la somme du travail accompli est impressionnante. Sa façon de travailler est simple mais efficace. M. Otis mobilise ceux qui l'entourent sur la solution d'un problème; il bâtit une requête, la fait signer et l'envoie à qui de droit, évêque ou ministre. Il aligne les raisons avec tant de logique qu'il est difficile pour celui qui la reçoit de répondre par un «non»; la chose est d'autant plus délicate que la multitude des signatures et des croix indique non pas le désir d'un seul homme mais une volonté collective.

Dès son arrivée au Saguenay, le nouveau curé se rend compte de deux choses: Saint-Alexis doit avoir son propre curé; de plus, il faut construire une nouvelle église à Saint-Alphonse. Il met donc immédiatement sur pied sa «machine-à-requêtes». Le 25 décembre 1856, les "paroissiens» demandent la permission de construire une nouvelle église. En juillet 1857, les «paroissiens» informent l'évêque que la construction de l'église va bientôt commencer et qu'il sera désormais impossible au curé 
de desservir Saint-Alexis; en octobre 1857, les «paroissiens» toujours, demandent de l'aide pour leur curé. Le 6 octobre 1857, ce dernier appuie la demande de ses paroissiens par une lettre personnelle. On croit comprendre qu'à la rigueur le curé de Saint-Alphonse se contenterait d'un vicaire qui pourrait se rendre à Saint-Alexis chaque dimanche; mais l'idéal, semble-t-il, serait que Saint-Alexis ait son propre curé; car (1) ils comptent sur des promesses...qui prennent du temps à se concrétiser; (2) le vice augmente; (3) La chapelle de Saint-Alphonse est trop petite; (4) On possède une chapelle bien organisée à Saint-Alexis. Le 16 mars 1858. M. Otis continue d'entrer le clou. Dans une nouvelle lettre, il parle de ces «malheureux orphelins de Saint-Alexis» qui sont toujours inquiets et il ajoute que leur «chapelle est encore suffisante pour contenir la population».

À force d'insistance et grâce à sa conviction profonde, à son pouvoir de persuasion et à ses talents d'organisateur, M. Otis réussit à se faire écouter de ses supérieurs; si bien que le 22 septembre 1858, M. LouisAntoine Martel arrive comme curé de Saint-Alexis et desservant des missions dont devait s'occuper M. Otis. Le premier juillet 1860 il écrit dans son journal qu'on est en train de creuser «les fondations de la nouvelle église de Saint-Alphonse».

M. Otis a réglé les deux problèmes majeurs qu'il avait trouvés à son arrivée. Mais il a dû en même temps vaquer à son travail pastoral ordinaire à Saint-Alphonse et à Saint-Alexis de même que dans les missions lointaines; il a trouvé le temps de construire des écoles et même de fonder la première bibliothèque de la région du Saguenay-Lac-Saint-Jean. Mais il a accompli tout cela à ses risques et périls. À tout moment, en effet, son journal nous informe de son état de santé précaire. Il parle de «maladie», de «fatigue», de «rhume», d' «indisposition», d' «enrouement», de «toux», d'«épuisement». Il se rend si bien au bout de ses forces que, brusquement, sans aucun avertissement, on trouve au 22 septembre 1861, la note suivante qui constitue la fin du troisième acte de cette vie trépidante d'un curé d'autrefois:

J'annonce mon départ de Saint-Alphonse en quelques mots. Je ne fus pas éloquent dans cette circonstance. - Je partis de St-Alphonse le 24 au soir; mais je ne fus rendu à l'Anse Saint-Jean que le 28 au soir. - Il fallut attendre à la Gde Baie dans l'exécrable bateau le temps favorable ${ }^{7}$.

On croirait lire une épître de saint Paul.

7 R. Lapointe, Le cahier de Monsieur Otis, Cahiers de Saguenayensia No 1 (Études et documents) 1986, p. 50. 


\section{L'Anse Saint-Jean}

Il existe, sur la rive sud du Saguenay, un village pittoresque qui, de nos jours, est devenu un endroit rêvé de villégiature: c'est L'Anse-SaintJean. C'est d'abord à cet endroit que s'arrêtèrent les pionniers lorsqu'ils vinrent à la conquête du Saguenay. Mgr Victor Tremblay nous dit à ce sujet: «C'est là qu'on a abattu le premier arbre quand la première équipe des hommes de la Société des Vingt-et-Un est venue au printemps de 1838 et le lieu n'a pas cessé d'être habité à partir de ce moment ${ }^{8}$ ».

Actuellement, L'Anse-Saint-Jean est dotée d'une excellente route, ses habitants sont à l'aise et coulent une existence paisible dans un décor de rêve; mais il y a une marge entre la vie menée par les premiers colons et celle des résidents ou des villégiateurs. La poésie du site reste toujours la même mais la vie quotidienne de cette époque héroïque était loin de posséder le charme et la douceur de celle d'aujourd'hui. Voici d'ailleurs ce que nous dit Arthur Buies à ce sujet:

Les colons de l'Anse Saint-Jean étaient pour ainsi dire des exilés. Il n'y avait point de voie de terre; en été, la voie d'eau seule existait par le Saguenay qui, l'hiver se couvrait d'un pont de glace et encore ce pont de glace refusait-il souvent passage des semaines entières à la suite de tempêtes de neige ou de vents violents qui rompaient et soulevaient la croûte et rendaient tout chemin impossible. (Le Saguenay et le bassin du LSJ, 1896, p. 123-124) ${ }^{9}$.

Arthur Buies cite un ancien curé de L'Anse- Saint-Jean, M. Adolphe Girard, qui, en 1871, écrivait:

La plus grande partie des habitants n'ont même pas le nécessaire, surtout le printemps. Ces pauvres gens vivent au jour le jour, et ils n'ont pas d'épargne pour le temps des semences. À cette époque, il faut gagner le pain pour la famille, et la saison favorable s'écoule de la sorte sans que l'on puisse suffisamment ensemencer les terres. Ici, ce sont les familles privilégiées qui ont de la viande toute l'année. (Ibid., p. 124) ${ }^{10}$.

M. Otis avait toujours eu un faible pour cette population dans le besoin et, même lorsqu'il fut déchargé de cette mission, il venait visiter ces pauvres. En quittant Saint-Alphonse, c'est là qu'il se rend exercer son ministère. En 1865, il rédige même des «Notes touchant les premiers établissements de L'Anse-Saint-Jean».

Son arrivée à cet endroit fut tout aussi tragique que son départ de Saint-Alphonse. Il note, en effet, dans son journal:

8 Victor Tremblay, Les trente aînées de nos localités, Publication de la Suciété historique du Saguenay No 19, 1968, p. 25.

9 A. Buies, Le Saguenay et le bassin du Lac Saint-Jean, 1896, p. 123.

10 Ibid., p. 124. 
Je suis arrivé dans la nuit du 28 septembre veille de Saint-Michel. N'ayant ni vin, ni hostie, je ne pus dire la messe le $29 .$. La lère messe. comme prêtre résident fut donc célébrée le 6 octobre fête du Saint-Rosaire ${ }^{11}$.

Puis, oubliant qu'il est épuisé, M. Otis se remet immédiatement à la tâche avec autant d'énergie qu'à Saint-Alphonse. Dès le 13 octobre 1861, le nouveau curé note dans son journal:

Parler des bâtisses. Étable et Sacristie doivent être prêts (sic) pour la Toussaint ${ }^{12}$.

Il recommence à composer des requêtes et à les faire signer. Le 2 mars 1862 , il note:

3 requêtes à signer: lère Bureau de poste; 2e Chaînage au Petit-Saguenay; $3 e$ Chemin à verbaliser dans le ler rang depuis Mézraïm jusqu'à la mer ${ }^{13}$.

Le 23 janvier 1865, il fonde une bibliothèque.

M. Otis n'avait pas seulement à s'occuper de L'Anse-Saint-Jean. Il lui fallait aussi desservir les missions environnantes. Dans son journal, il parle de L'Anse-à-l'eau, de Petit-Saguenay, de Rivière SainteMarguerite...

Avec une telle activité, la maladie continue ses ravages. M. Otis est un peu comme le vase brisé de Sully Prudhomme. La tuberculose est en train de pratiquer une sournoise meurtrissure dans cette nature trop ardente qui ne s'appartient pas. Permettez-moi de reproduire ici une lettre que $\mathbf{M}$. Dominique Racine, le futur évêque de Chicoutimi, écrivait à Mrr CharlesFrançois Baillargeon en 1866 à propos de M. Lucien Otis:

Que dirais-je à votre Grandeur de cette localité? Le pauvre et malheureux confrère qui dessert cette paroisse étant à se défendre constamment contre les attaques de la mort, ne peut guère penser et moins encore travailler à faire des améliorations. Mr. Otis use les restes de ses forces et de son courage à donner les offices obligés à ses paroissiens, même quand le triste état de sa santé exige qu'il demeure tranquille, dans sa maison. Un dimanche ou un jour de fête se présente-til, il se traîne à sa chapelle et commence un office qu'il n'est pas certain de terminer. Déjà, il lui est arrivé, pendant le cours d'un office, sentir ses forces l'abandonner et se faire apporter un siège pour se reposer. Plusieurs fois, je lui ai écrit l'invitant à venir se reposer ici., j’ai été chez lui croyant mieux réussir par ce moyen; mais je n'ai jamais pu le déterminer à s'éloigner de sa mission. Au commencement de juillet, il est venu passer quelques jours ici et chez les autres Messieurs, mais on voit que la crainte de mettre un ami à la gêne et celle de priver

11 R. Lapointe, Le Cahier de Monsieur Otis, Cahiers de Saguenayensia No 1, (Études et documents) 1986, p. 51.

12 Ibid., p. 52.

13 Ibid., p. 53. 
L'Anse-Saint-Jean de son curé le tient cloué à son presbytère. Si j'en crois le médecin, Mr. Otis ne passera certainement pas l'hiver sans succomber à sa maladie $^{14}$.

\section{Et ce fut la fin}

Le 6 septembre 1866, M. Lucien Otis fut remplacé par M. GeorgesÉric Sauvageau. Il se retira chez son frère Eucher à L'Anse-Saint-Jean et l'évêque lui conserva la juridiction qu'il possédait comme missionnaire de ce territoire. M. Sauvageau avait, lui aussi, une santé délicate. Il ne fut qu'un an comme curé de L'Anse-Saint-Jean. Quant à M. Otis, après plusieurs mois d'une convalescence qui semble avoir porté des fruits, il fut nommé assistant-principal de l'École normale Laval de Québec, à l'ouverture de l'année académique 1867-1868. Il ne fut que quelques mois à ce poste.

L'école normale Laval était une fondation récente. Son inauguration avait eu lieu le 12 mai 1857 et elle était logée dans le Vieux-Château; c'était une construction remontant à 1784 qui avait été érigée sous les ordres du gouverneur Haldimand dans le but de servir aux réceptions officielles et d'accueillir les personnages de la suite de sa Majesté britannique.

M. Lucien Otis ne semble pas avoir fait beaucoup de bruit à l'École normale Laval. L'ouvrage intitulé «Les noces d'or de l'École normale Laval» publié en 1908 parle de lui comme «ex-assistant principal» à la page 65, mais on laisse un carreau vide là où aurait dû être sa photo. C'est que durant ces derniers mois l'École normale Laval eut comme assistant principal un simple moribond qui s'éteignit lentement pour rendre l'âme le 12 juin 1868, à l'âge de 44 ans.

Ainsi, celui qui s'était identifié pendant dix ans avec le développement du Saguenay, qui avait laissé son nom à un lac poissonneux et à un canton en pleine expansion, s'éteignit sans attirer l'attention à l'ombre du Vieux-Château. Pourtant, parmi tous les personnages canadiens et britanniques qui avaient défilé dans ce monument prestigieux depuis la fin du dix-huitième siècle M. Otis, il est permis de le penser, était peut-être le plus grand.

Ce qu'a retenu l'Histoire concernant M. Lucien Otis, c'est l'image d'un prêtre zélé tout à son ministère, une âme de feu logée dans un vase fragile. Doux, humble, distingué et doué de talents artistiques, cet homme

14 Rapports sur les Missions du Diocèse de Québec et autres Missions qui en ont cidevant fait partie, 1839-1874. Semaine Religieuse de Québec, Vol. XIV, No 4, 14 septembre 1901 , p. 53. 
a, pendant dix ans, usé sa santé au service de la région du Saguenay. Les documents qui nous sont parvenus et les témoignages laissés par les contemporains mettent l'accent sur ses nombreuses qualités dont deux émergent: douceur et dévouement.

Au moment où les colons sont dépourvus d'instruction et travaillent de l'aube à la nuit pour être capables de survivre; au moment où les structures sociales sont réduites à leur plus simple expression, le «Bon Monsieur Otis» est là pour animer la vie spirituelle, sans doute, mais aussi pour jouer un rôle de suppléance dans différents secteurs. Avocat, ii piaide la cause des plus démunis; juge, il arbitre les conflits; notaire, il rédige des contrats qu'il s'agisse de la vente d'un animal ou de l'acquisition d'un lot; architecte, il dresse des plans de chapelles ou de presbytères. Comme ses confrères-prêtres œuvrant dans le milieu, M. Otis est le seul qui soit assez instruit pour établir un lien entre les pauvres colons et les autorités civiles ou religieuses et qui soit assez détaché des biens de ce monde pour inspirer confiance de part et d'autre. Partout où il pose le pied, M. Otis laisse des traces profondes dans la terre saguenéenne. Il ne passe que cinq années à Saint-Alphonse mais cela est suffisant pour que Pierre-Georges Roy puisse dire: «ll fit beaucoup pour la colonisation de ce canton ${ }^{15}$ ». Il se transporte ensuite à L'Anse-Saint-Jean où il passe le même nombre d'années; il améliore tellement ce pauvre coin perdu que l'abbé LouisAntoine Martel sent le besoin de dire: «Mon bon voisin M. L. Otis qui prend tant d'intérêt pour L'Anse (sic) et à qui est dû certainement presque tout l'accroissement que cette nouvelle place a pris depuis quelques années ${ }^{16}$ ». Qu'on ne se surprenne pas si l'on a voulu, en guise d'auréole et de remerciement, donner le nom de M. Otis à un canton, à un lac, et, plus tard, à une paroisse.

Profondément attaché à sa famille et à la région du Saguenay, «Monsieur Otis» souhaitait reposer dans la terre saguenéenne à côté de sa mère, comme il le laissait entendre à son évêque ${ }^{17}$. Au lieu de quitter sa paroisse aussitôt après avoir été remplacé, il poursuit sa convalescence à L'Anse-Saint-Jean durant une année encore. Il sent sa fin prochaine et, comme sa mère repose à L'Anse-Saint-Jean il pourra ainsi réaliser son rêve d'aller la rejoindre. Mais voilà que sa vigueur fait un dernier sursaut. À contre-cœur sans doute et par obéissance il prend alors le chemin de Québec. Après avoir traîné durant quelques mois encore sa pénible existence, il s'éteignit enfin tel un exilé sur une terre étrangère.

15 Pierre-Georges Roy, Les noms géographiques de la Province de Québec, Lévis, 1906, p. 294.

16 Lettre de Louis-Antoine Martel à Mgr de Tloa, 18 octobre 1860.

17 OTIS, lettre à son évêque. 
Tandis qu'à Québec il disparaît sans bruit comme la flamme d'une lampe qui vient d'épuiser son huile; tandis qu'à Sainte-Anne-de-la-Pocatière, on a conservé peu de traces de cet élève calme et studieux, dans la région du Saguenay, témoin de son dévouement, il a laissé derrière lui et malgré lui un sillage qui refuse de disparaître. Les cartes de notre région ont immortalisé son nom en lui faisant don à la fois d'un lac, d'une paroisse et d'un canton. En attendant que le Dictionnaire biographique du Canada joigne sa voix à la sienne dans un concert de reconnaissance, le Saguenay répète à l'intention de Monsieur Otis ces paroles de Gilles Vigneault:

J'ai pour toi un lac quelque part au monde Un beau lac tout bleu

Comme un œil ouvert sur la nuit profonde Un cristal frileux

Qui tremble à ton nom comme tremble feuille

À brise d'automne et chanson d'hiver ${ }^{18}$.

18 Gilles Vigneault, Avec les vieux mots, Éditions de l'A R C, 1964, p. 62. 


\section{Bibliographie}

Buies, Arthur, Le Saguenay et le Bassin du Lac Saint-Jean, Léger Brousseau, 1896.

Carrière, Gaston, (OMI) Histoire documentaire de la congrégation des missionnaires oblats de Marie-Immaculée dans l'Est du Canada. Tome II: Les Oblats au Saguenay. Éditions de l'Université d'Ottawa, 1959, p. 271-337.

Keiso, Samuei J. Noies on ine Sagueriany for touirists and others, Québec, Morning Chronicle, 1862, 31 p. Traduit en français par Raoul Lapointe: Saguenayensia avril-juin 1988, p. 7-21.

Lapointe, Raoul, Le cahier de Monsieur Otis, Cahiers de Saguenayensia No 1, (Études et documents) 1986, $76 \mathrm{p}$.

Malchelosse, Gérard, Généalogie de la Famille Otis, branche canadienne, Montréal G. Ducharme, 1921, 86 p.

Martel, Louis-Antoine, Notes sur le Saguenay, Texte manuscrit publié par le Centre d'Études et de Recherche historiques du Saguenay, Séminaire de Chicoutimi, 1968.

Noces d'or de l'École normale Laval, Laflamme et Proulx, Québec, 1908.

Pérez, Michel, Charles Trenet, Collection Poètes d'aujourd'hui, 1964.

Pilote, François, Le Saguenay en 1851, Histoire du passé, du présent et de l'avenir probable du Haut-Saguenay au point de vue de la colonisation, Québec, A. Côté, 1852.

Rapports sur les Missions du Diocèse de Québec et autres Missions qui en ont ci-devant fait partie, 1839-1874. Semaine religieuse de Québec, vol. XIV, 14 septembre 1901, p. 53.

Revue Canadienne (La), Vol. 29 (1893) et Vol. 30 (1894). Le Fort et le Château Saint-Louis (Ernest Gagnon).

Saguenayensia, Juillet-Septembre 1983, Trois témoins des temps héroïques, par Raoul Lapointe, p. 59-72.

Tremblay, Victor, Histoire du Saguenay, depuis les origines jusqu'en 1870. Publication de la Société historique du Saguenay, No 21, 1968, (Édition nouvelle).

Tremblay, Victor, Les trente aînées de nos localités. Brefs historiques. Publication de la Société historique du Saguenay, No 19, 1968.

Vigneault, Gilles, Avec les vieux mots, Éditions de l'A R C, Québec, 1964. 